Discussion Similar to what has been observed in lupus-prone mice and IL- $2 \%$ mice, Treg from SLE patients show the classical hallmarks of IL-2 deficiency with loss of CD25 expression and impaired homeostasis. Our in vitro results show that these Treg defects can be restored by low-dose IL-2 treatment, suggesting IL-2 as a novel therapeutic target for SLE.

\section{A9.15 REMISSION AS THE MAIN THERAPEUTIC TARGET: COMPARATIVE EFFICACY OF FOUR TREATMENT REGIMENS IN EARLY RHEUMATOID ARTHRITIS (RA) PATIENTS (PTS)}

doi:10.1136/annrheumdis-2013-203223.15

E Fedorenko, G Lukina, Y Sigidin. Laboratory of Clinical Pharmacology, Research Institute of Rheumatology RAMS, Moscow, Russian Federation

Background Early RA contains the "the window of opportunities" for achieving the best results of therapy including remissions. It is very important to determine the influence of different treatment regimens on the remission rate in patients (pts) with early RA.

Objectives To compare development of remissions in pts with early ( $<2$ years duration) RA who were randomly assigned to receive four different strategies of DMARDs treatment.

Methods One hundred forty-one pts with RA of less than 2 years duration (122 women, mean age 51 years, mean disease duration 24 weeks, mean DAS28 5.9; 64\% RF-positive, 59\% ACCP-positive) were randomised to receive one of the following treatment regimens: methotrexate (MTX, up to $20 \mathrm{mg} /$ week, $35 \mathrm{pts}$ ); MTX plus prednisolone (P) $10 \mathrm{mg}$ daily (MTX-P, 34 pts); MTX-P plus methylprednisolone (MP) $1000 \mathrm{mg}$ intravenously on the first day of treatment (MTX-P-MP, 35 pts); leflunomide 20 mg daily (LEF, 37 pts). Duration of treatment was one year. Efficacy of therapy was assessed by EULAR criteria. Control points were 3, 6 and 12 months from the beginning of therapy. The primary endpoint was the development of remission. Results At baseline all groups were comparable in their demographic, clinical and radiographic characteristics. One hundred twenty-seven pts completed the study. By the $3 \mathrm{~d}$ month in the MTX group only $3.1 \%$ of the patients reached clinical remission, while in the combination groups of MTX with GC (including MP intravenously) $21.3 \%$ and $28.6 \%$, respectively. By the 6 th month the same tendency continued: combination of MTX with GC showed the greatest frequency of remissions $-33.3 \%$ and $23.5 \%$, respectively. In the MTX and LEF monotherapy groups the corresponding figures were $15.2 \%$ and $20.6 \%$, respectively. By the 12 th month the frequency of remissions was significantly higher in pts treated with the combination of MTX and GC, including high doses of MP (37.5\% and $29.4 \%$, respectively) than in the MTX and LEF monotherapy groups (11.4\% and $16.2 \%$, respectively).

Conclusions In pts with early RA combined treatment with MTX and GC led to the significantly higher remission rate as compared with MTX and LEF monotherapy.

\section{A9.16 SYNOVIAL FIBROBLASTS FROM PATIENTS WITH RHEUMATOID ARTHRITIS DIFFERENTIATE INTO DISTINCT FIBROBLAST SUBSETS IN THE PRESENCE OF CARTILAGE}

doi:10.1136/annrheumdis-2013-203223.16

${ }^{1}$ Adam P Croft, 'Amy Naylor, ${ }^{2}$ Birgit Zimmermann, 'Debbie Hardie, 'Guillaume Desanti, ${ }^{1}$ Maria Jaurez, ${ }^{2}$ Ulf Muller-Ladner, ${ }^{1}$ Andrew Filer, ${ }^{2}$ Elena Neumann, ${ }^{1}$ Christopher Buckley. ${ }^{1}$ Centre for Translational Inflammation Research, University of Birmingham, Birmingham, UK; 'Internal Medicine and Rheumatology Justus-Liebig-University Gießen, KerckhoffKlinik, Bad Nauheim, Germany

Background Rheumatoid arthritis synovial fibroblasts (RASF) migrate to distant tissue sites and damage articular cartilage. Using novel markers of RASF subsets to identify lining and sub-lining layer RASF we investigated the ability of RASF to undergo selfassembly, transmigration and cartilage degradation in vivo.
Methods Healthy human cartilage was co-implanted subcutaneously into the flank of SCID mice together with RASF. On the contralateral flank, cartilage was implanted without cells. After 60 days, implants and blood were analysed. Human cells were detected using immunohistochemistry for species-specific antibodies. For in vitro studies, RASF were isolated from patients with established RA and healthy controls and the expression of cellular markers were defined.

Results RASF at the ipsilateral implant differentiated into distinct fibroblast subsets in the presence of cartilage. Cells proximal to cartilage expressed markers of a lining layer phenotype (GP38, FAP, VCAM-1 and Cadherin-11). These cells attached to, invaded and degraded cartilage. Cells more distal to cartilage expressed sublining layer phenotype markers including CD248 and CD90. Cells expressing CD248 and CD90 were never observed in the lining layer (proximal to cartilage) and never invaded cartilage. The development of this stromal architecture mirrored that observed in vivo in the inflamed synovial membrane. This stromal pattern of distinct lining layer and sub lining layer differentiation was recapitulated in the contralateral implant that contained only cartilage. In addition, we demonstrate that RASF in vitro can be directed towards either a lining layer (GP38, FAP, VCAM-1 and Cadherin-11) or sub-lining layer phenotype (CD248 and CD90) following cytokine treatment. The lining layer, but not sub lining cell phenotype is associated with increased cartilage degradation in vitro.

Conclusions RASF have an activated cell phenotype ex vivo. In vitro and in vivo they display plasticity with the capacity to differentiate into distinct cell sub-populations that morphologically distinguish between the lining and sub-lining layer of the human joint. In vivo cell sub-population differentiation occurs locally at the site of engraftment and recapitulates the lining anatomy observed at the site of origin. This phenomenon is dependent on the release of tissue factors found present in the joint microenvironment following cartilage damage. This inflammatory microenvironment is required for cartilage destruction by RASF. Cellular therapies targeting RASF specific subsets are an unexplored but important therapeutic target to modulate inflammation and may provide an avenue to minimise joint damage in patients with RA.

\section{A9.17 TARGETING THE EXPRESSION OF MIR-146A IN MOUSE INFLAMMATORY Ly6C high MONOCYTE SUBSET FOR THERAPEUTIC INTERVENTION IN ARTHRITIS}

doi:10.1136/annrheumdis-2013-203223.17

1,2M Ammari, 1,2] Duroux-Richard, 1,2J Presumey, ${ }^{3} \mathrm{C}$ Roubert, 4,5V Escriou, ${ }^{1,2,6} \mathrm{C}$ Jorgensen, 1,2,6 F Apparailly. ${ }^{1}$ INSERM, U 844, 80 rue Augustin Fliche, F-34295 Montpellier, France, ${ }^{2}$ UniversityofMedicine, $F-34000$ Montpellier, France, ${ }^{3}$ ExploratoryUnit, Sanofi-AventisR R D, 371 rue duProfesseurJBlayac, 34184Montpellier, France, ${ }^{4} I N S E R M$, U 1022, UFR des Sciences Pharmaceutiques et Biologiques; ' Avenue de l'Observatoire, 75006 Paris, France, ${ }^{5}$ CNRS, UMR8151, 75006 Paris, France; ${ }^{6}$ University Hospital of Montpellier, Clinical Unit for Osteoarticular Diseases, F-34295 Montpellier, France

Background and Objectives Monocytes can give rise to different cell types including osteoclasts (OC), which play an important role in maintaining bone homeostasis by resorbing bone matrix. Circulating monocytes consist of at least two main functional subsets of immune cells, Ly6Chigh and Ly6C low monocytes, arising from a common progenitor in the bone marrow. Excessive and prolonged activation of inflammatory LyC6 $6^{\text {high }}$ monocytes is a hallmark of many inflammatory diseases including arthritis. Among key molecular rheostats, micro (mi) RNAs are a class of regulatory RNAs that control basic biological functions and orchestrate inflammatory responses. Among master miRNAs of innate immunity, miR-146a exerts a negative retro-control on inflammation transduction signals and inhibits osteoclastogenesis. Despite aberrant increased expression in rheumatoid arthritis (RA), miR-146a is unable to properly down regulate inflammation, leading to prolonged TNF 